\title{
SOFTWARE CLÍNICO PARA EL ANÁLISIS TEMPORAL Y ESPECTRAL DE REGISTROS DE ELECTROCARDIOGRAFÍA
}

\section{SOFTWARE CLINICAL FOR ANALYSIS TEMPORAL AND SPECTRAL OF ELECTROCARDIOGRAPHY RECORDS}

\author{
PhD. J. L. Ramón Valencia*. PhD. A. García-Sánchez**. PhD. J. Roca-Dorda**** \\ * Universidad El Bosque, Bogotá Colombia \\ Grupo de Investigación. OSIRIS Programa de Bioingeniería, \\ leninramon@unbosque.edu.co \\ ****** Universidad Politécnica de Cartagena, Cartagena, España \\ ${ }^{2}$ Grupo de Investigación. Modelos y sistemas para procesado de señales \\ y series Temporales, Astronomía y Fiabilidad de sistemas. \\ ***Grupo Investigación. EIMED.
}

\begin{abstract}
Resumen: El trabajo investigativo se basó en el desarrollo y validación de una herramienta informática de uso clínico y de bajo costo para los neurólogos. Este Bioinstrumento virtual, se implementó en un Hospital General Universitario de Alicante (HGUA), en donde se aplican los protocolos para un test de pruebas autónomas, con el fin de construir y adquirir un banco de señales electrocardiográficas, luego son procesadas en un software denominado, "Sistema de Procesado Digital de Parámetros del ECG" (SPDPECG), para la extracción de los parámetros en el dominio del tiempo y de la frecuencia. Por último se hace un análisis estadístico de las variables para diferenciar entre los grupos controles y de Enfermos de Párkinson.
\end{abstract}

Palabras clave: Bio-instrumento Virtual, Electrocardiografía y pruebas autónomas.

\begin{abstract}
The research was based on an informatics tool of clinical use and low cost for neurologist. This virtual bio-instrument was implemented on the Alicante University General Hospital (HGUA), where some protocols were applied for an autonomy practice test with the purpose to build and acquire an electrocardiographic database that are later processed on an established software. "ECG Parameters Digital Processing System (SDPECG), for the extraction of the parameters on the frequency and time domain. At last, a statistical analysis of the variables was done in order to distinguish between the control groups and the patients under Parkinson category.
\end{abstract}

Keywords: Virtual bio-instrument, electrocardiography and autonomic testing.

\section{INTRODUCCIÓN}

Los avances alcanzados en la Cardiología, se deben a la incorporación de herramientas diagnósticas basadas en la informática médica, según estudios realizados se encontró que el Sistema Nervioso Autónomo (SNA) se evalúa a través de test de índices clínicos:
- Test de respiración profunda realizada en reposo del paciente (Ewing D.J. et al., 1984).

- Maniobra de Válsala respirando en forma controlada.

- Ejercicio Isométrico estando de pie sin moverse. 
- Respuesta de la presión arterial al ortostatismo desarrollada caminando de forma usual (Ewing D.J. et al., 1984).

De acuerdo con lo anterior, podemos decir que hay estudios, en donde se evidencia la afectación precoz del SNA en la Enfermedad del Parkinson (EP) y Parkinsonismos (Kallio et al., 2000), (Haapaniemi et al., 2001) Figura 1. En particular, existe una interacción entre SNA y el corazón, el cual funciona como sistema realimentado a través del Sistema Nervioso Central (SNC), en donde se envían estímulos y son utilizados por el SNA, para dar repuesta ante un fenómeno que altera la frecuencia cardiaca en el corazón a través del nodo sinusal ó marcapasos, estas alteraciones varían y se ven reflejadas en un test de comparación del ritmo cardiaco. Según la literatura, los cambios de actividad permiten al corazón obtener fluctuaciones en la frecuencia cardiaca, de tal forma que influyan en el SNA, ya sea a nivel del sistema nervioso simpático SNP, en donde el ritmo cardiaco se hace más lento, ocasionando una Bradicardia, mientras que en el sistema nerviosos parasimpático SNP, el ritmo cardiaco se acelera ocasionando, el ritmo cardiaco oscila entre 90 a 110 pulsaciones por minuto. Asimismo, existe un patrón que modula los dos sistemas cardiacos nombrados anteriormente, se llama Variabilidad de la Frecuencia Cardiaca VFC, que se define como la variación del periodo de la señal ECG con el tiempo (Marco V, José et al., 2014). Figura 2.

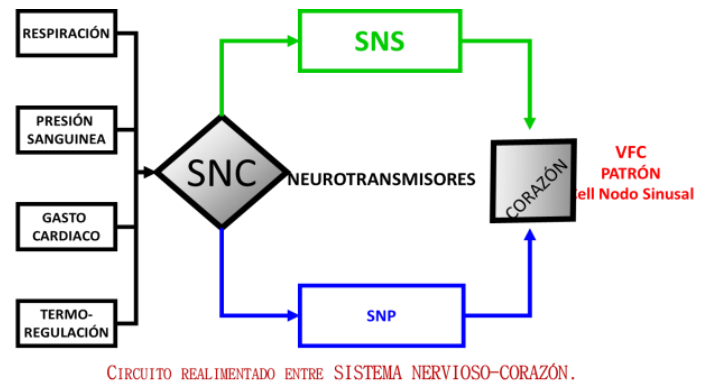

Fig. 1. Circuito realimentado Sistema NerviosoCorazón.

\section{METODOLOGÍA}

Los métodos estándar para la establecer la VFC se obtuvieron implementado una herramienta software (SPDP-ECG) para la obtención de parámetros en domino del tiempo y de la frecuencia en (ep) y (co), (García-Sánchez A. et al., 2006), (García-Sánchez A. et al., 2007)
Las fuentes de extracción de la señal ECG, para el procesado digital de señales son de datos de los pacientes del Hospital General Universitario de Alicante (HGUA), la extracción de los datos se hizó a partir de un Holter digital de marca SYNEFLASH con una frecuencia de muestreo de $220 \mathrm{~Hz}$. Luego son aplicados protocolos de auscultación que comprenden: Respiración profunda, consiste en una prueba Valsalva, 30:15 Índice, el ejercicio isométrico y respuesta de la presión arterial a orto-estático. Para efectuar la evaluación de estas pruebas, se tuvieron en cuenta los criterios de (Ewing et al., 1984), para finalizar se le hace firma un consentimiento informado para la extracción de los datos.

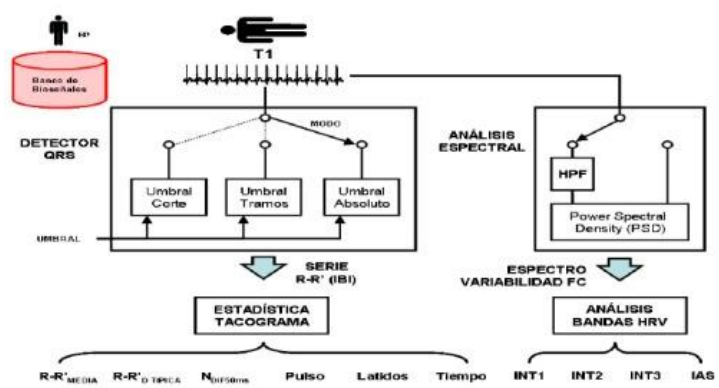

Fig. 2. Diagrama operativo de la herramienta software (SPDP-ECG).

El funcionamiento de la herramienta (SPDPECG). En primer lugar, la señal es preprocesada, para que el software aplique un filtro pasa bajo, para eliminar todo tipo de interferencias producidas por los electrodos (ruidos y triboleléctricos dinámicos). Posteriormente es acondicionada la señal, y se procede a detectar la FC por medio de tres tipos de umbrales: "umbral de corte", "umbral de tramos" y "umbral absoluto" (Moreno at al., 2013).

En cada caso se aplican tres algoritmos diferentes, seleccionando un simple botón, para obtener la VFC. Una vez se tiene seleccionado el tipo de algoritmo que mejor se adapta a la señal ECG, se extraen las variables en el dominio del tiempo relacionadas: R-R "media, R-R" desviación típica y el Número de la Diferencia (número de pulsos con una duración superior a 50 milisegundos en todo el tramo).

Además, se obtienen variables de tipo control: como son la duración media por minuto de las pulsaciones (lo que se conoce como pulso) (latidos/minuto), la duración total del registro y los latidos totales en el registro. 
Por último, se aplican en el dominio de la frecuencia por medio de la FFT (Transformada Rápida de Fourier) para obtener los parámetros en la estimación de Densidad Espectral de Potencia (PSD) asociadas a las bandas valores "a muy bajas frecuencias" INT1 $(0-0.05 \mathrm{~Hz})$, "banda a baja frecuencia" INT2 $(0.05-0.15 \mathrm{~Hz})$ y las "alta frecuencia" INT3 $(0.15-0.40 \mathrm{~Hz})$ (Ramón et al., 2012).

\section{RESULTADOS Y DISCUSIÓN}

El software clínico, está desarrollada en un programa de National Instruments versión LabView 13. La herramienta ofrece al especialista una interface gráfica con el usuario, en donde le permite interactuar con otras ventanas como son: Extracción de los datos por el programa (guardar parámetros en el dominio del tiempo y frecuencia), espectrocardiograma completo, parámetros de programa; absolutos, relativos y promediados según intervalos de frecuencia, Análisis espectral, Segmento obtenido de una señal; Variabilidad de Frecuencia Cardiaca (figura 3).

Además, el software clínico (SPDP-ECG), permite extraer los datos numéricos en archivos txt, proporcionando unas variables de tipo cualitativo y cuantitativo. Para la extracción de los datos se utilizó las muestras ECG de larga duración, procedentes de Hospital General Universitario de Alicante (HGUA), se clasifican según las categorías (ep) (10) y (co) (13) para un total de 23 pacientes.

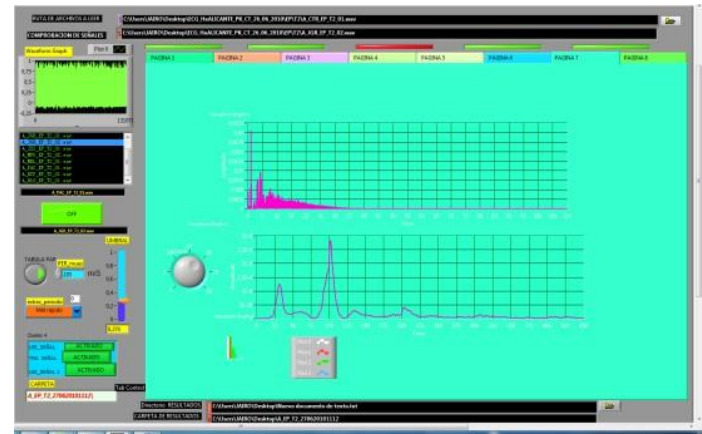

Fig. 3. Análisis espectral en el dominio de frecuencias aplicando FFT a una señal ECG.

Dentro de las dificultades del estudio, se destacan el número reducido de pacientes incluidos y la necesidad de que el mismo paciente no tenga enfermedades cardiovasculares; en general que pueda afectar al sistema autónomo en particular. Sin embargo, esta última condición es una de las que probablemente permitió obtener resultados más favorables en cuanto a la densidad espectral de potencia.

\section{CONCLUSIONES}

Se ha realizado una revisión bibliográfica de la enfermedad de parkinson y la ECG, lo que nos ha permitido abordar el diseño de un sistema de análisis computarizado. La aplicación software desarrollada, bajo entorno de LabView permite procesar la señal ECG y extraer los parámetros adecuados para su caracterización. La aplicación permite extraer toda la información necesaria para realizar las estimaciones pertinentes.

\section{AGRADECIMIENTOS}

Al Dr. A. Monge-Argilés, neurólogo del Hospital General Universitario de Alicante (HGUA) por la adquisición y evaluación de los datos de los pacientes.

\section{REFERENCIAS}

Ewing D.J., J.M.M. Neilson and P. Travis. (1984). "New method for assessing cardiac parasympathetic activity using 24 hour electrocardiograms", British Heart Journal, vol. 52, pp. 396-402.

Kallio M, Haapaniemi TH, Turkka K, Suominen K, Tolonen U, Sotamiemi H, Heikkila VP, Myllya V. (200). Heart rate variability in patients untrated parkinson`s disease. Eur J Neurol; 7:667-672.

Haapaniemi TH, Pursiainen v, Korpelainen JT, Huikuri HV, Sotaniemi KA, Myllyla VV. (2001). Ambulatory ECG and analysis of heart rate variability in Parkinson's disease. J Neurol Neurosurg Psychiatry; 70 (3): 30531dsd.

García-Sánchez A, J Roca-Dorda, JL RamónValencia, J Roca-González, A Monge, M Ortega. (2006). Computer Based Tool for Temporal and Spectral Analysis of Electrocardiographic Records. Computers in Cardiology; 33 pp: 585-588.

Larre Claudia, Oscar I., Marco V. Sistema de Análisis de la Variabilidad de la Frecuencia Cardiaca, Grupo de Biología Teórica, Instituto de Investigaciones Biomédicas. at: http://www.researchgate.net/publication/26 7217566.

García Sánchez A, J.L. Ramón-Valencia, A. Monge Ardiles, A. Guillamón Frutos, J. Roca-Dorda y J 
Roca-González. (2007). "Sistema de ayuda, análisis e interpretación de la modificación de la variabilidad de la frecuencia cardiaca (VFC) en Enfermos de Parkinson y parkinsonismos" XXV Congreso Anual de la Sociedad Española de Ingeniería Biomédica, CASEIB 2007, Cartagena los días 14, 15 y 16 de Noviembre de 2007.

Moreno Rubio J., Jiménez López A, Barrera Lombana N. (2013). El amplificador de potencia de carga sintonizada. Revista colombiana de tecnologías de Avanzada. Vol. 2, No. 22, pp. 9-13
Valencia Ramón, J. L. (2012). Herramienta basada en el ordenador para análisis temporal y espectral de registros ECG: aplicación a la enfermedad de Parkinson, Tesis-Univ. Politécnica de Cartagena, Departamento de Tecnología Electrónica, Fecha de Publicación, 20 de enero de 2012, 251 p. 